\title{
REAL ISOMORPHIC COMPLEX BANACH SPACES NEED NOT BE COMPLEX ISOMORPHIC
}

\author{
J. BOURGAIN
}

\begin{abstract}
It is shown that complex Banach spaces may be isomorphic as real spaces and not as complex spaces. If $X$ is a complex Banach space, denote $\bar{X}$ the Banach space with same elements and norm as $X$ but scalar multiplication defined by $z \cdot x=\bar{z} \cdot x$ for $z \in \mathbf{C}, x \in X$. If $X$ is a space of complex sequences, $\bar{X}$ identifies with the space of coordinate-wise conjugate sequences and its norm is given by $\|x\|_{\bar{X}}=\|\bar{x}\|_{X}$, where $\bar{x}=\left(\bar{z}_{1}, \bar{z}_{2}, \ldots\right)$ for $x=\left(z_{1}, z_{2}, \ldots\right)$. Obviously $X$ and $\bar{X}$ are isometric as real spaces. In this note, we prove that $X$ and $\bar{X}$ may not be linearly isomorphic (in the complex sense). The method consists in constructing certain finite dimensional spaces by random techniques.
\end{abstract}

1. Introduction. In considering isomorphisms of complex Banach spaces, a natural question is whether or not real isomorphic spaces are complex isomorphic. A theorem due to S. Mazur and S. Ulam asserts that two complex Banach spaces which are isometric as metric spaces are necessarily linearly isometric as real normed spaces [8]. The purpose of this paper is to exhibit a complex space $X$ with the property that $X$ and $\bar{X}$, clearly real-isometric, may not be even complex isomorphic. In this setting, the result also appears to be of interest to representation theory. The method used to construct the space $X$ is essentially based on finite dimensional techniques. More precisely, $X$ will be an $l^{2}$-direct sum $X=\bigoplus_{2} X_{k}$, where the $X$ are suitably built finite dimensional spaces. Hence $X$ is reflexive and from construction it will follow that $X$ has type $p$ and cotype $q$ whenever $p<2<q$ (see [7] for definitions and generalities). The first step to obtain the $X_{k}$ will be to consider certain random norms on $\mathbf{C}^{N}$. The present and earlier work (cf. $[\mathbf{4}, \mathbf{9}]$ ) show that this "random" technique is an easy and effective way to obtain finite dimensional normed spaces with various pathologies.

It should be said that the isometric version of the problem, i.e. the existence of real isometric noncomplex-isometric spaces, was solved previously by N. Kalton [6]. Related results can be found in a recent preprint of S. Szarek [10]. He shows, among other things, that in the finite dimensional setting one may have the extremal situation $d(Y, \bar{Y})=O(\operatorname{dim} Y)$, where $d$ stands for Banach-Mazur distance.

The paper contains two further sections. In $\S 2$ it is explained how the infinite dimensional problem reduces to a finite-dimensional one, namely, the construction of the spaces $X_{k}$. Computations are carried out in $\S 3$. Since in many aspects our approach is a repetition of a known reasoning, we may be quick on certain points (it may be helpful for the reader to consult the Appendix of [9]).

Received by the editors July 16, 1984 and, in revised form, February 26, 1985.

1980 Mathematics Subject Classification. Primary 46B20, 46B10, 46A45. 
2. Reduction to a finite dimensional problem. For each $n$, we consider a complex $n$-dimensional Banach space $Y=Y_{n}$ with norm satisfying

$$
|x| \leq\|x\|_{Y} \leq \sqrt{n}|x| \quad\left(x \in \mathbf{C}^{n}\right),
$$

where $|x|=\left(\sum\left|x_{j}\right|^{2}\right)^{1 / 2}$ denotes the $l^{2}$-norm on $\mathbf{C}^{n}$.

This space $Y$ will be obtained by a random method. More precisely, the extreme points of the unit ball of $Y$ will be $K$ randomly choosen points on the sphere $S^{n-1}=\left\{x \in \mathbf{C}^{n},|x|=1\right\}$. As usual in such construction, $\log k \sim \log n$ and (1) may be assumed automatically satisfied.

The space $\bar{Y}$ has norm defined by $\|x\|_{\bar{Y}}=\|\bar{x}\|_{Y}$, denoting $\bar{x}=\sum_{j=1}^{n} \bar{x}_{j} e_{j},\left\{e_{j}\right\}_{j=1}^{n}$ $=$ unit vectors. Let $\left\{n_{k}\right\}$ be a rapidly increasing sequence of positive integers and $0<\theta_{k}<1$ a sequence converging to 0 . At this point, we do not explicite conditions since they will be clear to the reader from what follows.

For each $k$, define the real interpolation space (cf. [2]) $X_{k}=\left[l_{n_{k}}^{2}, Y_{n_{k}}\right]_{\theta_{k}, 2}$. (Instead of the real method, we may have used the complex interpolation method as well.) Let $X$ be the $l^{2}$-direct sum $X=\bigoplus_{2} X_{k}$ for $k=1,2, \ldots$. Hence, we have the identifications

$$
\bar{X}=\bigoplus_{2} \bar{X}_{k} \quad \text { with } \bar{X}_{k}=\left[l_{n_{k}}^{2}, \bar{Y}_{n_{k}}\right]_{\theta_{k}, 2}
$$

The next lemma is basically known (see $[\mathbf{1}$, p. 77] for instance).

LEMMA 1. Let

$$
\frac{1}{p}=\frac{1-\theta}{2}+\frac{\theta}{1}, \quad \frac{1}{p}+\frac{1}{q}=1 .
$$

Then $\left[l_{n}^{2}, Y_{n}\right]_{\theta, 2}$ has type $p$ and cotype $q$.

PROOF. Since the dual space of $\left[l_{n}^{2}, Y_{n}\right]_{\theta, 2}$ identifies with $\left[l_{n}^{2}, Y_{n}\right]_{\theta, 2}$, the cotype property follows from the type property and the type-cotype duality for spaces with type. Let us denote by $\left\{\varepsilon_{i}\right\}$ a finite Rademacher sequence. The map $\left\{x_{i}\right\}_{i=1}^{n} \stackrel{\alpha}{\rightarrow}$ $\sum \varepsilon_{i} x_{i}$ has the obvious boundedness properties

$$
l^{2}\left(l_{n}^{2}\right) \rightarrow L^{2}\left(l_{n}^{2}\right), \quad l^{1}\left(Y_{n}\right) \rightarrow L^{2}\left(Y_{n}\right) .
$$

(These notations refer to the vector valued $l^{p}$ and $L^{p}$-spaces.)

By the Lions-Peetre interpolation theorem (see [2, p. 121]), $\left[l^{2}\left(l_{n}^{2}\right), l^{1}\left(Y_{n}\right)\right]_{\theta, 2}$ identifies with the Lorentz-space $l^{p, 2}\left(\left[l_{n}^{2}, Y_{n}\right]_{\theta, 2}\right)$. Since the identity map

$$
l^{p}\left(\left[l_{n}^{2}, Y_{n}\right]_{\theta, 2}\right) \rightarrow l^{p, 2}\left(\left[l_{n}^{2}, Y_{n}\right]_{\theta, 2}\right)
$$

is norm decreasing, it follows that $\alpha$ is

$$
l^{p}\left(\left[l_{n}^{2}, Y_{n}\right]_{\theta, 2}\right) \rightarrow L^{2}\left(\left[l_{n}^{2}, Y_{n}\right]_{\theta, 2}\right)
$$

bounded. Hence the type $p$ property of $\left[l_{n}^{2}, Y_{n}\right]_{\theta, 2}$ is shown.

LEMMA 2. Given a positive integer $d$, there is $\theta>0$ such that the BanachMazur distance $d\left(E, l_{d}^{2}\right) \leq 2$ for any d-dimensional subspace $E$ of $\left[l_{n}^{2}, Y_{n}\right]_{\theta, 2}$.

Proof. The known estimate (see [3, p. 84])

$$
d\left(E, l_{d}^{2}\right) \leq T_{p}(E) C_{q}(E) d^{2(1 / p-1 / q)}
$$

$\left(T_{p}, C_{q}\right.$ referring to type and cotype constants respectively) and Lemma 1 indeed imply that $d\left(E, l_{d}^{2}\right) \leq d^{\theta}$. 
As a consequence of this lemma, we may state

COROllary 3. A sequence $\left\{\theta_{j}\right\}$ may be found such that for each $j$, any $2 n_{j}$ dimensional subspace of $\bigoplus_{2}\left(\sum_{k>j} X_{k}\right)$, similarly $\bigoplus_{2}\left(\sum_{k>j} \bar{X}_{k}\right)$, is 2-Hilbertian. Moreover, $\theta_{j}$ depends only on $n_{j-1}$.

Remember that $n_{j}=\operatorname{dim} X_{j}$. The sequence $\left\{\theta_{j}\right\}$ converges to 0 and is defined such that subspaces of the "tale" $\bigoplus_{k>j} X_{k}$ of dimension $\sum_{k \leq j} n_{k}$ are Hilbertian. This aspect of the construction is used in the following

LemMa 4. Assume $T$ is a linear isomorphism between the spaces $X, \bar{X}$. Then for each $j$, the inequality

$$
d\left(X_{j} \oplus l_{n}^{2}, \bar{X}_{j} \oplus l_{n}^{2}\right) \leq C n_{j-1}\|T\|\left\|T^{-1}\right\|, \quad\left(m=n_{j}\right)
$$

has to hold.

PROOF. For fixed $j$, the space $Z=\operatorname{span}\left\{T\left(X_{k}\right) \cup \bar{X}_{k} ; k=1, \ldots, j\right\}$ is clearly of the form $Z=\bigoplus_{k<j} \bar{X}_{k} \oplus \bar{X}_{j} \oplus V$, where $V$ is a subspace of $\bigoplus_{k>j} \bar{X}_{k}$, and $\operatorname{dim} V \equiv$ $R \leq \sum_{k \leq j} n_{k}$. By construction, $T^{-1}(Z)=\bigoplus_{k<j} X_{k} \oplus X_{j} \oplus W$ with $W$ a subspace of $\bigoplus_{k>j} X_{k}, \operatorname{dim} W=R$, From Corollary 3 , we get for $m^{\prime}=\sum_{k<j} n_{k}+R<m+4 n_{j-1}$ (assuming $n_{k}$ rapidly increasing)

$$
\begin{aligned}
d\left(T^{-1}(Z), X_{j} \oplus l_{m^{\prime}}^{2}\right) & \leq \sum_{k<j} d\left(X_{k}, l_{n_{k}}^{2}\right)+d\left(W, l_{R}^{2}\right) \\
& \leq \sum_{k<j} n_{k}^{1 / 2}+2
\end{aligned}
$$

and a similar estimate for $d\left(Z, \bar{X}_{j} \oplus l_{m^{\prime}}^{2}\right)$. Consequently

$$
d\left(X_{j} \oplus l_{m}^{2}, \bar{X}_{j} \oplus l_{m}^{2}\right) \leq C n_{j-1}\|T\|\left\|T^{-1}\right\|
$$

as announced.

What has to be obtained is now clear. In order to contradict the conclusion of Lemma 4, it will suffice to show that for a fixed $0<\theta<1$ and for $n$ chosen large enough, the interpolation space $\left[l_{n}^{2}, \bar{Y}_{n}\right]_{\theta, 2}$ with $Y_{n}$ the random space described above, cannot be obtained as a uniform quotient space of $\left[l_{n}^{2}, Y_{n}\right]_{\theta, 2} \oplus l_{n}^{2}$ (uniformly relative to $n \rightarrow \infty)$. This fact will be verified in the next section.

Actually, the argument applied to prove this yields also a minoration of the form $d\left(Y_{n}, \bar{Y}_{n}\right)>c n / \log n$. In [10], a more delicate argument leads to $d\left(Y_{n}, \bar{Y}_{n}\right)=$ $O(n)$, thus the order of the diameter of the $n$-Minkowski compactum (cf. [4]).

3. Estimations related to the spaces $Y_{n}$. Let $0<\theta<1$ be fixed as well as $n>n(\theta, M)$, where $M$ is any number. Denote for convenience

$$
B=\left[l_{n}^{2}, Y\right]_{\theta, 2}, \quad \bar{B}=\left[l_{n}^{2}, \bar{Y}\right]_{\theta, 2} .
$$

The space $Y$ is determined by a random choice of $K=n^{C}$ points $\xi_{1}, \ldots, \xi_{K}$ in the unit sphere $S=S^{n-1}$ of $\mathbf{C}^{n}$, which will be the extreme points of the unit ball of $Y$. Denote also $S_{K}$, the $K$-fold product $S \times \cdots \times S$ equipped with its product measure $\mathbf{P}_{S_{K}}$. Fix a constant $M$. Our purpose is to verify that for $n>n(\theta, M)$ large enough, an estimation of the order $e^{-\sqrt{K}}$ for the probability $(*)$

$$
\mathbf{P}_{S_{K}}\left[\|u\|_{B \rightarrow \bar{B}} \leq M,\|v\|_{l_{n}^{2} \rightarrow \bar{B}} \leq M ; \xi_{j} \in\left\{u(x)+v(y) ;\|x\|_{B} \leq 1,|y| \leq 1\right\}, \forall_{j}\right]
$$


relative to a choice $\left(\xi_{1}, \ldots, \xi_{K}\right) \in S_{K}$, is valid. Here $u: \mathbf{C}^{n} \rightarrow \mathbf{C}^{n}$ and $v: \mathbf{C}^{n} \rightarrow \mathbf{C}^{n}$ is any fixed pair of linear maps.

Taking $K=n^{5}$, say, a standard argument (cf. [9]) involving nets of linear operators $u, v$ will then lead to the existence of a system $\xi_{1}, \ldots, \xi_{K}$ and thus a space $Y$ with following property: If $\|y\|_{B \rightarrow \bar{B}} \leq M$ and $\|v\|_{l_{n}^{2} \rightarrow \bar{B}} \leq M$, then $u+v: B \oplus l_{n}^{2} \rightarrow \bar{B}$ is not a 1-quotient map in the sense that $\exists z \in \bar{B},\|z\|_{\bar{B}} \leq 1$ and $z$ not of form $z=u(x)+v(y),\|x\|_{B} \leq 1,|y| \leq 1$. With respect to the previous section, the role of $M$ will be $C n_{j-1}$ and $n=n_{j}$. So it remains to evaluate (*).

Besides some complications due to the fact that $B$ was defined as an interpolation space, let us now indicate two features distinguishing these computations from Gluskin's argument [4].

(1) The role of the conjugation when comparing $B, \bar{B}$ or $Y, \bar{Y}$. this conjugation will be exploited using the well-known fact that $\alpha+\beta, \alpha-\beta$ are independent, whenever $\alpha, \beta$ are independent Gaussian processes.

(2) The lack of independence in evaluating certain probabilities will require some elementary martingale theory.

We start to analyze (*). Notations $c>0, C<\infty$ indicate numerical constants.

LEMMA5. Either there exists an orthogonal projection $P$ in $\mathbf{C}^{n}$ of rank $\left[\frac{n}{2}\right]$ such that $\|P u\|_{l_{n}^{2} \rightarrow l_{n}^{2}} \leq 2 M$ or $(*)<e^{-c K}$.

Proof. Notice that since the unit ball of $\bar{B}$ is contained in the Euclidean ball,

$$
\|u\|_{B \rightarrow \bar{B}} \geq\|u\|_{B \rightarrow l_{n}^{2}} \geq\|u\|_{Y \rightarrow l_{n}^{2}}=\sup _{1 \leq j \leq k}\left|u\left(\xi_{j}\right)\right| .
$$

Considering the polar decomposition of $u$, it is easy to see that with probability at least $1-e^{-c K}$, the latter quantity will dominate $2 M$ as soon as more than $\frac{n}{2} s$ numbers of $u$ are dominated by $6 M$. The existence of the required projection $P$ is then immediate.

Next, we claim $v$ may be assumed to fulfill

$$
\int_{S} \sup _{|x| \leq 1}|\langle v(x), P \bar{\xi}\rangle| d \xi<n^{-\theta / 3} .
$$

Indeed

LEMMA 6. If (2) does not hold, then $\|v\|_{l_{n}^{2} \rightarrow \bar{B}}>M$ whenever $\left(\xi_{1}, \ldots, \xi_{K}\right)$ is $S_{K}$ (taking $n$ large enough).

Proof. By construction, $\|x\|_{(\bar{B})^{*}} \leq|x|^{1-\theta}\|x\|_{(\bar{Y})^{*}}^{\theta}$ and hence

$$
\begin{aligned}
\int_{S} \sup _{|x| \leq 1}|\langle v(x), P \bar{\xi}\rangle| d \xi & \leq\|v\|_{2 \rightarrow \bar{B}} \int_{S} \sup _{\|x\|_{\bar{B}} \leq 1}|\langle x, P \bar{\xi}\rangle| d \xi \\
& =\|v\|_{2 \rightarrow \bar{B}} \int_{S}\|P \bar{\xi}\|_{(\bar{B})^{*}} d \xi \\
& \leq\|v\|_{2 \rightarrow \bar{B}} \int_{S} \sup _{1 \leq j \leq K}\left|\left\langle P \bar{\xi}, \bar{\xi}_{j}\right\rangle\right|^{\theta} d \xi \\
& \leq[(\log K) / n]^{\theta / 2}\|v\|_{2 \rightarrow \bar{B}} .
\end{aligned}
$$

Remembering that $K=n^{C}$, the lemma follows. 
It follows from construction of $P$ (see Lemma 5) that for $|\xi| \leq 1$

$$
\begin{aligned}
\sup _{\|x\|_{B} \leq 1}|\langle u x, P \bar{\xi}\rangle| & =\left\|u^{*} P \bar{\xi}\right\|_{B^{*}} \leq\left|u^{*} P \bar{\xi}\right|^{1-\theta}\left\|u^{*} P \bar{\xi}\right\|_{Y^{*}}^{\theta} \\
& \leq(2 M)^{1-\theta} \sup _{1 \leq j \leq K}\left|\left\langle\bar{\xi}, P u \xi_{j}\right\rangle\right|^{\theta} .
\end{aligned}
$$

Going back to $(*)$, assume $\bar{\xi}_{j}=u(x)+v(y)$, where $\|x\|_{B} \leq 1,|y| \leq 1$.

$$
\left|\left\langle\bar{\xi}_{j}, P \bar{\xi}_{j}\right\rangle\right| \leq(2 M)^{1-\theta}\left\{\left|\left\langle\bar{\xi}_{j}, P u \xi_{j}\right\rangle\right|^{\theta}+\sup _{k \neq j}\left|\left\langle\bar{\xi}_{j}, P u \xi_{k}\right\rangle\right|^{\theta}\right\}+\sup _{|y| \leq 1}\left|\left\langle v(y), P \bar{\xi}_{j}\right\rangle\right| .
$$

It will suffice to show that with probability at least $1-e^{-K^{1 / 2}}$, some $j=1, \ldots K$ will violate (4).

From the fact that $\int_{S}|\langle\bar{\xi}, P \bar{\xi}\rangle| d \xi>\frac{1}{2}$ and inequality (2), it follows that with probability $>1-e^{-c K}$ for at least $K / 2$ values in the sequence $\left(\xi_{1}, \ldots, \xi_{K}\right)$ in $S$,

$$
\left|\left\langle\bar{\xi}_{j}, P \bar{\xi}_{j}\right\rangle\right|>\frac{1}{4}
$$

and

$$
\sup _{|y| \leq 1}\left|\left\langle v(y), P \bar{\xi}_{j}\right\rangle\right|=o(1)
$$

The first two terms in (4) remain to be analyzed. The term $\left\langle\bar{\xi}_{j}, P u \xi_{j}\right\rangle$ is the crucial one. For $\xi \in S=\left\{\sum_{t=1}^{n} z_{t} e_{t} \mid z_{t} \in \mathbf{C}\right.$ and $\left.\sum\left|z_{t}\right|^{2}=1\right\}$, with $\xi=x+i y$ with $x, y \in \mathbf{R}^{n},|x|^{2}+|y|^{2}=1$. Thus we identify $S$ with the real sphere $S^{2 n-1}$. Then,

$$
\begin{aligned}
&\langle\bar{\xi}, P u \xi\rangle=\langle x-i y, P u x+i P u y\rangle \\
&=\langle x, P u x\rangle-\langle y, P u y\rangle-i\langle y, P u x\rangle-i\langle x, P u y\rangle, \\
&|\langle\bar{\xi}, P u \xi\rangle| \leq|\langle x+y, P u(x-y)\rangle|+2|\langle x, P u y\rangle|+2|\langle y, P u x\rangle| .
\end{aligned}
$$

Since the map $(x, y) \rightarrow((x+y) / \sqrt{2},(x-y) / \sqrt{2})$ is a measure preserving transformation of $S^{2 n-1}$, it follows

$$
\int_{S}|\langle\bar{\xi}, P u \xi\rangle| d \xi \leq C \int_{S^{2 n-1}}|\langle x, P u y\rangle| d(x, y)<C / \sqrt{n}
$$

Hence, again with probability $1-e^{-c K}$, most of the $j$-values will satisfy

$$
\left|\left\langle\bar{\xi}_{j}, P u \xi_{j}\right\rangle\right|<C n^{-1 / 2} \text {. }
$$

The last contributions $\left|\left\langle\bar{\xi}_{j}, P u \xi_{k}\right\rangle\right|$ for $j \neq k$ have to be evaluated. The independence problem will be taken care of by relying on Azuma's distributional inequality for martingales with square summable increments.

LEMMA 7. Let $\left\{d_{k}\right\}$ be a martingale difference sequence on a probability space $(\Omega, \mathbf{P})$ and denote $s=\left(\sum\left\|d_{k}\right\|_{\infty}^{2}\right)^{1 / 2}$. then

$$
\mathbf{P}\left[\left\|\sum d_{k}\right\|>\lambda\right]<\bar{e}^{c \lambda^{2} / s^{2}} .
$$

The result is well known and easy to prove (see [5], for instance, for a proof). 
Define for each $j$ the function $a_{j}$ (resp. $b_{j}$ ) of $\xi_{1}, \ldots, \xi_{j-1}\left(\operatorname{resp} . \xi_{j+1}, \ldots, \xi_{K}\right)$

$$
a_{j}=\int_{S} \sup _{k<j}\left|\left\langle\bar{\xi}, P u \xi_{k}\right\rangle\right| d \xi ; \quad b_{j}=\int_{S} \sup _{k>j}\left|\left\langle\bar{\xi}, P u \xi_{k}\right\rangle\right| d \xi
$$

satisfying $a_{j}, b_{j}<c((\log K) / n)^{1 / 2}$. Application of Lemma 7 with $\Omega=S_{K}$ and the natural product-structure yields

$$
\mathbf{P}_{S_{K}}\left[\sum_{1 \leq j \leq K}\left\{\sup _{k<j}\left|\left\langle\bar{\xi}_{j}, P u \xi_{k}\right\rangle\right|-a_{j}\right\}>\lambda\right]<e^{-c \lambda^{2} / K}, \quad(\lambda>0),
$$

$$
\mathbf{P}_{S_{K}}\left[\sum_{1 \leq j \leq K} \sup _{k<j}\left|\left\langle\bar{\xi}_{j}, P u \xi_{k}\right\rangle\right|>K n^{-1 / 3}\right]<e^{-K n^{-2 / 3}}
$$

and similarly, inverting the order,

$$
\mathbf{P}_{S_{K}}\left[\sum_{1 \leq j \leq K} \sup _{k>j}\left|\left\langle\bar{\xi}_{j}, P u \xi_{k}\right\rangle\right|>K n^{-1 / 3}\right]<e^{-K n^{-2 / 3}}
$$

Combining (8) and (9) we see that with probability $>1-e^{-c K^{1 / 2}}(K$ was choosen large enough), most of the $j$ will verify

$$
\sup _{k \neq j}\left|\left\langle\bar{\xi}_{j}, P u \xi_{k}\right\rangle\right| \leq n^{-1 / 4} .
$$

It results from previous considerations (5), (6), (7) and (10), that with probability $>1-e^{-c K^{1 / 2}}(4)$ will fail for some $j=1, \ldots, K$ ( $n$ has to be taken sufficiently large w.r.t. $\theta$ ). Hence the proof is completed.

\section{REFERENCES}

1. B. Beauzamy, Espaces d'interpolation réels: Topologie et géométrie, Lecture Notes in Math., vol. 666, Springer-Verlag, Berlin and New York, 1978.

2. J. Bergh and J. Löfstrom, Interpolation spaces: an introduction, Gundlehren der Mathematischen Wissenschaften, vol. 223, Springer-Verlag, Berlin and New York, 1976.

3. T. Figiel, V. D. Milman and J. Lindenstrauss, The dimension of almost spherical sections of convex bodies, Acta Math. 139 (1977), 53-94.

4. E. D. Gluskin, The diameter of the Minkowski compactum is roughly equal to n, Functional Anal. Appl. 15 (1981), 72-73.

5. W. B. Johnson and G. Schechtman, Embedding $l_{p}^{n}$ into $l_{1}^{n}$, Acta Math. 149 (1982), 77-85.

6. N. Kalton, unpublished.

7. J. Lindenstrauss and L. Tzafriri, Classical Banach spaces. II, Vol. 97, Springer-Verlag, Berlin and New York, 1979.

8. S. Mazur and S. Ulam, Sur les transformations isometriques d'espaces vectoriels normes, C. R. Acad. Sci. Paris 194 (1932), 946-948.

9. S. J. Szarek, the finite dimensional basis problem with an appendix on nets of Grassmann manifolds, Acta Math. 151 (1983), 153-179.

10. preprint.

Department of Mathematics, Vrije Universiteit Brussels, Pleinlaan 2-F7, 1050-BRUSSELS

Institut Hautes Etudes SCIEnCES, BuR-SUR Yvette, France 